\title{
Comparison of Enantioselective CEC Separation of OT-MIP Capillary Columns with Templates of Various Camphor Derivatives Made by the Pre-established General Preparation Protocol
}

\author{
Shabi Abbas Zaidi, Seung Mi Lee, Ju Young Lee, and Won Jo Cheong* \\ Department of Chemistry, Nano Fine Center, and Institute of Basic Research, Inha University, Incheon 402-751, Korea \\ ${ }^{*}$ E-mail: wjcheong@inha.ac.kr \\ Received July 12, 2010, Accepted August 5, 2010
}

\begin{abstract}
Some open tubular (OT) molecule imprinted polymer (MIP) silica capillary columns with templates of camphor derivatives such as 10-camphorsulfonic acid (10-CSA), 10-camphorsulfonamide (10-CS) and camphor-p-tosyl hydrazone $(\mathrm{CTH})$ have been successfully prepared by the prior generalized preparation protocol. The three MIP thin layers of different templates showed quite different morphologies. The chiral selectivity of each MIP column for the template enantiomers was optimized by changing eluent composition and $\mathrm{pH}$. The optimization conditions were found to be different for the three MIPs. This work suggests prospective successful extension of the generalized preparation protocol for OT-MIP silica capillary columns toward templates of a variety of chemical groups.
\end{abstract}

Key Words: Camphor derivatives, Chiral separation, Molecule imprinted polymer, Generalized preparation protocol, Morphology

\section{Introduction}

Molecular imprinting technology involves an effective method for the fabrication of polymer with cavities of a template and the polymer is subsequently capable of selectively rebinding the template. ${ }^{1-3}$ In the last decade, this technique has shown wide application in pharmaceuticals, environmental chemistry and chromatographic separation. ${ }^{4-5}$ Although molecularly imprinted polymers (MIPs) have shown great potential in HPLC based analysis, however, the use of MIP coupled with CEC is still trying to make its niche in analytical science..$^{6-8}$

Although the MIP preparation methodology seems relatively easy, optimization of MIP formulation to achieve the best results makes it complicated. The best MIP can only be realized by empirical optimization via synthesis and evaluation of various polymers. Furthermore, a very different MIP preparation protocol is generally required to resolve a new pair of enantiomers. The entire procedure is time consuming and needs to be generalized. In one report, the cross selectivity synthesized by using only one imprint showed chiral separation for several pairs of $\beta$-blockers. ${ }^{9}$ In one of our earlier reports, we showed that long open tubular molecularly imprinted columns (OT-MIP) in capillary can offer exceptionally high separation efficiencies for ketoprofen. ${ }^{10}$ Recently, we demonstrated a generalized preparation protocol of OT-MIP columns for a number of templates

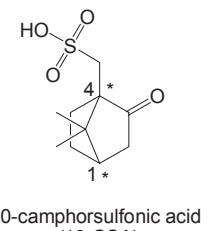

(10-CSA)

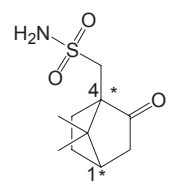

10-camphorsulfonamide (10-CS)

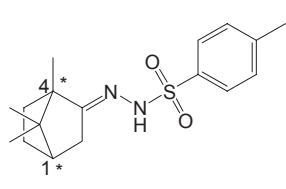

camphor-p-tosylhydrazone (CTH) including non-steroidal anti inflammatory drugs (NSAIDs) in CEC. ${ }^{11}$ The S-camphor MIP capillary column prepared by the generalized formulation, however, was unable to resolve racemic camphor in the same study.

Camphor is a terpenoid, used as preservative in pharmaceutical and cosmetics. Besides, it is being considered as an excellent plasticizer for explosives. It is usually sold as racemate, however. U.S.P specifies the d-form. ${ }^{12}$ Chiral separation of 10-camphorsulfonic acid has been carried out by a bovine serum albumin (BSA) immobilized zirconia column. ${ }^{13}$ The authors suggested that there were several interactions such as hydrophobic, electrostatic, hydrogen bonding as well as charge transfer processes playing vital roles in chiral separation. Although the retention times were small, the resolution was quite satisfactory. In another report, a baseline resolution was achieved for 10-camphorsulfonamide with a commercially available Chiralpak AD column in LC-MS separation. ${ }^{14}$

Despite of aforementioned chiral separations, not many studies have been devoted for the direct enantioseparation of camphor derivatives. In our knowledge, this is the first report ever concerning simultaneous chiral separation of 10-camphorsulfonic acid (10-CSA), 10-camphorsulfonamide (10-CS) and camphor- $p$-tosyl hydrazone (CTH) on OT-MIP columns. The molecular structures of the templates are given in Fig. 1. All the columns were prepared by the generalized formulation reported in our prior study. ${ }^{11}$ We have obtained rather satisfactory chiral separation with the OT-MIP capillary columns in this study, thus expanding the application of the generalized preparation protocol for OT-MIP capillary columns to a variety of templates seems promising.

\section{Experimental}

Chemicals and materials. Fused silica capillaries (50 $\mu \mathrm{m}$ ID, study. The chiral center is denoted by an asterisk. 
$365 \mu \mathrm{m}$ OD) were purchased from Alltech (Deerfield, IL, USA). Methacrylic acid (MAA), ethylene glycol dimethacrylate (EDMA), $\gamma$-methacryloxypropyl trimethoxysilane ( $\gamma$-MAPS), 4-styrenesulfonic acid (4-SSA), glacial acetic acid, sodium acetate, (1S)-10-camphorsulfonic acid, (1S)-10-camphorsulfonamide, $(1 S)$-camphor- $p$-tosyl hydrazone $(\mathrm{CTH}),(1 R)$-10camphorsulfonamide and $(1 R)$-camphor- $p$-tosyl hydrazone and racemic 10-camphorsulfonic acid were obtained from SigmaAldrich (St. Louis, MO, USA). Azobisisobutyronitrile (AIBN) was purchased from Junsei Chemical (Tokyo, Japan). HPLC grade acetonitrile and water were obtained from SK Chemicals (Ulsan, Korea). All of the reagents except for 4-SSA (sodium salt hydrate) were used as received. The acidification of 4SSA was carried out as reported in our previous study. ${ }^{3}$

Instrumentation. Electrochromatographic experiments were performed with an Agilent (Waldbronn, Germany) $\mathrm{HP}^{3 \mathrm{D}} \mathrm{CE}$ system with a diode array detector and the Chemstation data processing software. The electrolytes were prepared by titrating $\mathrm{NaH}_{2} \mathrm{PO}_{4}$ or $\mathrm{Na}_{2} \mathrm{HPO}_{4}$ with $\mathrm{H}_{3} \mathrm{PO}_{4}$ or $\mathrm{NaH}_{2} \mathrm{PO}_{4}$, to the desired $\mathrm{pH}$ within the range of $\mathrm{pH} 2.5$ - 8.0. The buffers were then added to acetonitrile in the proportions desired. All analytical samples were made to a concentration of $2.0 \mathrm{mg} / \mathrm{mL}$ by dissolving in the mobile phase and stored in a refrigerator. All the samples and eluents were filtered through a $0.2 \mu \mathrm{m}$ cellulose membrane before analysis. Samples were injected hydrodynamically for $6 \mathrm{~s}$ under a pressure of $8 \mathrm{mbar}$. The detection wavelength was set to $214 \mathrm{~nm}$. All the separations were carried out at a constant CE voltage of $25 \mathrm{kV}$ and a temperature of $25^{\circ} \mathrm{C}$ throughout. Each newly installed OT-MIP column was flushed with running the eluent for about $1 \mathrm{hr}$ to acquire the stable baseline and flushed with the same eluent for $5 \mathrm{~min}$ between consecutive analyses. The effective length of all the MIP columns was $28 \mathrm{~cm}$ (total length; $36.4 \mathrm{~cm}$ ).

Fabrication of open tubular $\boldsymbol{S}$-enantiomer MIP capillary columns with various template molecules. The fused silica capillary was modified as previous studies. ${ }^{3,8,10-11}$ Briefly, the silica capillary was treated with $\mathrm{NaOH}$, water, $\mathrm{HCl}$, water, and acetone in sequence, and dried under $\mathrm{N}_{2}$. A solution composed of $4 \mu \mathrm{L} \gamma$-MAPS in $1 \mathrm{~mL}$ of $6 \mathrm{mM}$ acetic acid was filled in the capillary for $6 \mathrm{~h}$ and the capillary was flushed with methanol and dried under $\mathrm{N}_{2}$.

Thin film of each MIP was fabricated inside the capillary as reported earlier by our group ${ }^{3}$ except that the template was replaced with the same molar amount of template enantiomer of 10-camphorsulfonic acid (10-CSA), 10-camphorsulfonamide (10-CS) or camphor- $p$-tosyl hydrazone (CTH). Each capillary was filled with the degassed and sonicated monomer mixture composed of template molecule $(0.020 \mathrm{mM})$, MAA $(8.2 \mu \mathrm{L})$, EDMA $(59 \mu \mathrm{L}), 4-\mathrm{SSA}(2 \mathrm{mg})$ and AIBN $(3.5 \mathrm{mg})$ dissolved in $1 \mathrm{~mL} \mathrm{9/1} \mathrm{(v/v)} \mathrm{acetonitrile/} \mathrm{2-propanol,} \mathrm{by} \mathrm{a} \mathrm{sy-}$ ringe installed with a $0.2 \mu \mathrm{m}$ syringe filter. The capillary was sealed and kept in a water bath at $50{ }^{\circ} \mathrm{C}$ for $4 \mathrm{~h}$. After polymerization, the capillary column was thoroughly rinsed with methanol-acetic acid (85/15 v/v \%) and acetonitrile in sequence. A small piece of capillary tip was cut for SEM characterization. A detection window was created at a distance of $8.4 \mathrm{~cm}$ from the outlet end of each capillary column by removing the polyimide coating.

\section{Results and Discussion}

In the following part, we will discuss the influences of molecular structures of different templates on variable architectures of morphology resulted from the OT-MIP columns. Furthermore, a systematic investigation was carried out for the chiral separation performance by varying the various chromatographic parameters such as eluent composition and $\mathrm{pH}$ of eluent.

Molecularly imprinted polymer morphology. Figure 2 Shows the SEM cross-sectional views of OT-MIP capillary columns constructed with (1S)-10-camphorsulfonic acid, (1S)-10-camphorsulfonamide and $(1 S)$-camphor $p$-tosyl hydrazone, respectively. As indicated in Figure 2, variances of MIP layer morphology were observed. According to Gonzalez and coworkers, the reason for different morphologies secured by different polymerization conditions may be attributed to the different templates' binding capacity, specificity, as well as chemical and thermal characteristics during the formation of polymer network. $^{15}$

Comparing the molecular structures of Figure 1, the basic

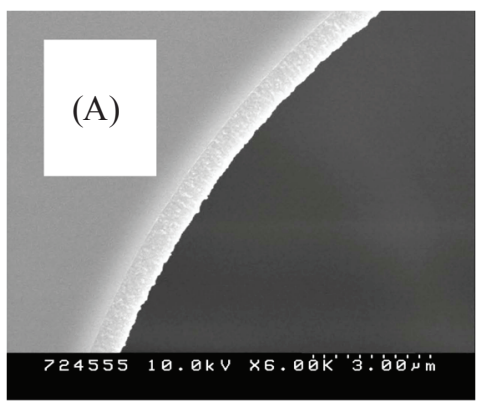

10-Camphorsulfonic acid

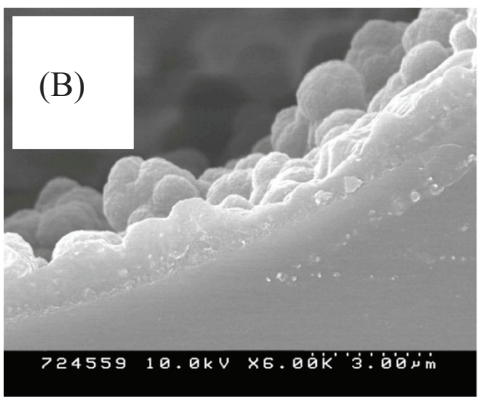

10-Camphorsulfonamide

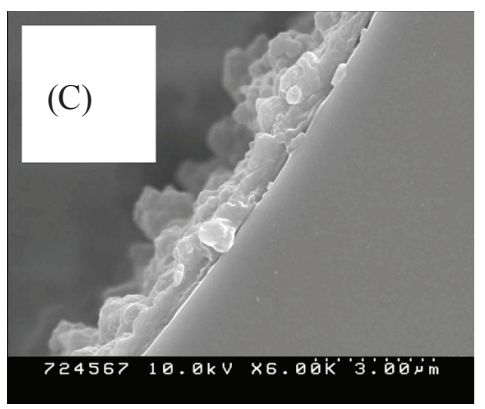

Camphor-p-tosylhydrazone

Figure 2. The cross-sectional SEM views of various OT-MIP columns with different templates. (A) (1S)-10-Camphorsulfonic acid, (B) (1S)10-Camphorsulfonamide, (C) (1S)-Camphor- $p$-tosyl hydrazone. 
Table 1. Column separation efficiency and resolution data of the OT-MIP columns under optimized eluent conditions

\begin{tabular}{ccccc}
\hline MIP template (analyte) & optimized eluent $^{a}$ & $\mathrm{~N}_{\mathrm{r}} / \mathrm{m}^{b}$ & $\mathrm{~N}_{\mathrm{s}} / \mathrm{m}^{c}$ & Resolution $^{d}$ \\
\hline 10-Camphorsulfonic acid & $(92 / 08, \mathrm{pH}-3.0)$ & $59500 \pm 1500$ & $24000 \pm 800$ & $1.2 \pm 0.03$ \\
10-Camphorsulfonamide & $(70 / 30, \mathrm{pH}-6.0)$ & $252000 \pm 7900$ & $100800 \pm 4200$ & $10.6 \pm 0.20$ \\
Camphor- $p$-tosyl hydrazone & $(92 / 08, \mathrm{pH}-7.0)$ & $65400 \pm 1300$ & $38300 \pm 600$ & $3.4 \pm 0.06$ \\
\hline
\end{tabular}

${ }^{a}$ Mixing volume $\%$ (acetonitrile $v$ s. buffer) and $\mathrm{pH}$ of $50 \mathrm{mM}\left(\mathrm{NaH}_{2} \mathrm{PO}_{4}+\mathrm{Na}_{2} \mathrm{HPO}_{4}\right)$ buffer. ${ }^{b}$ Number of theoretical plates/meter for $R$-enantiomer. ${ }^{c}$ Number of theoretical plates/meter for $S$-enantiomer. ${ }^{d}$ Calculated by the half height method.

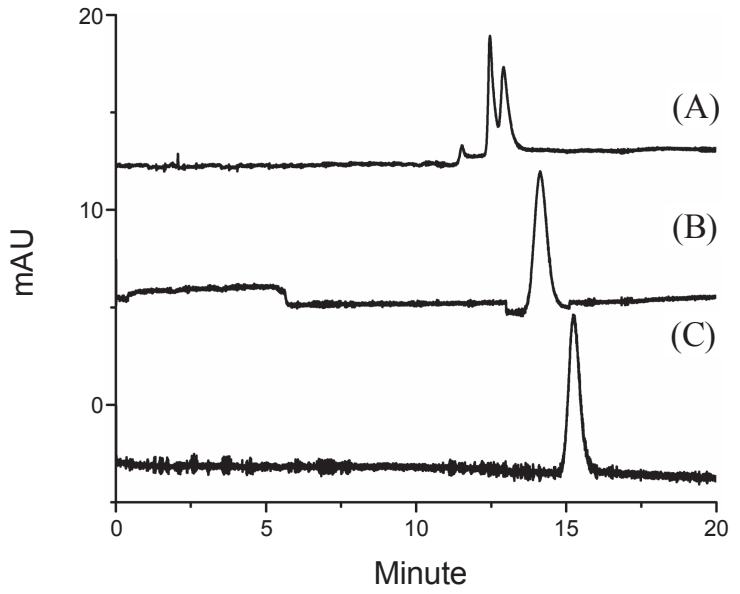

Figure 3. Effect of acetonitrile content on chiral separation of racemic 10-camphorsulfonic acid by the (S)-10-CSA MIP. (A) 92\%, (B) 80\%, (C) 70\%. First peak; (1R)-10-Camphorsulfonic, Second peak; (1S)-10Camphorsulfonic acid. Mobile phase: $\mathrm{ACN} / 50 \mathrm{mM}$ sodium dihydrogen phosphate (pH: 3.5), applied voltage: $30 \mathrm{KV}$, temperature: $25^{\circ} \mathrm{C}$, injection: $8 \mathrm{mbar} / 6 \mathrm{sec}$.

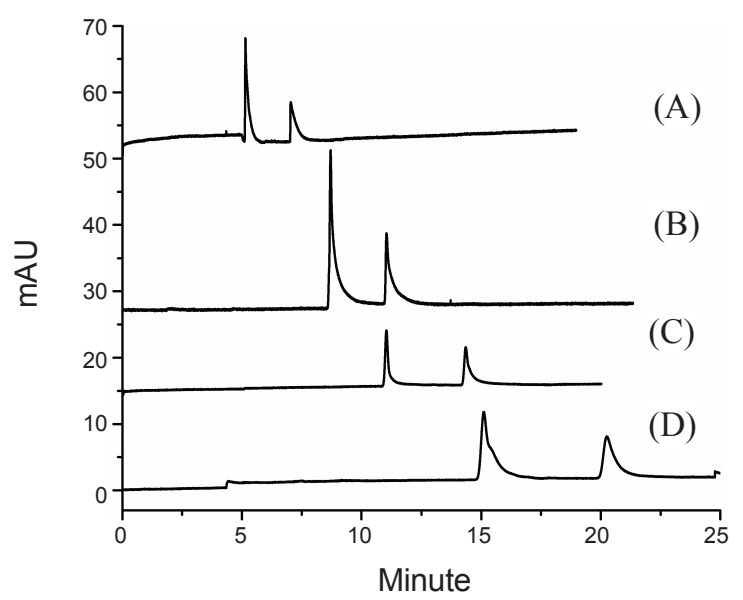

Figure 4. Effect of acetonitrile content on chiral separation of racemic 10-camphorsulfonamide by the (S)-10-CS MIP. (A) $92 \%$, (B) $80 \%$, (C) 70\%, (D) 60\%. First peak; (1R)-10-Camphorsulfonamide, Second peak; (1S)-10-Camphorsulfonamide. Mobile phase: ACN/50mM $\mathrm{NaH}_{2} \mathrm{PO}_{4} / \mathrm{Na}_{2} \mathrm{HPO}_{4}$ (pH: 7.0). Other conditions are the same as in Figure 3.

structure of the three templates is the same except for the substituent attached to the parent structure. The camphor skeleton has two chiral centers at position 1 and 4 (denoted as asterisks in Figure 1), but they are tightly connected with three chains in the camphor structure, thus there are only 2 enantiomers for

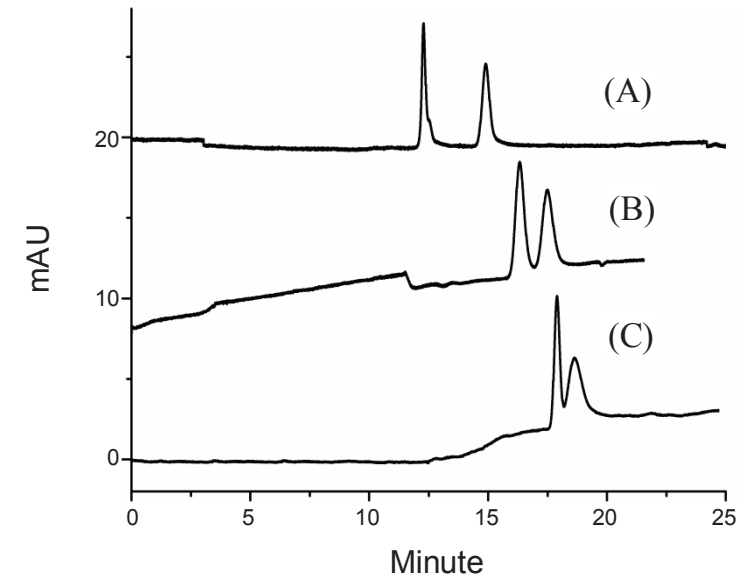

Figure 5. Effect of acetonitrile content on chiral separation of racemic camphor-p-tosyl hydrazone by the (S)-CTH MIP. (A) $92 \%$, (B) $80 \%$, (C) 70\%. First peak; $(1 R)$-Camphor- $p$-tosyl hydrazone, Second peak; (1S)-Camphor- $p$-tosyl hydrazone. Mobile phase: $\mathrm{ACN} / 50 \mathrm{mM} \mathrm{NaH}_{2}-$ $\mathrm{PO}_{4} / \mathrm{Na}_{2} \mathrm{HPO}_{4}$ (pH: 7.0). Other conditions are the same as in Figure 3.

each molecule of Figure 1 owing to such particularly bound structure. The chrial center of position 1 is spatially more accessible than that of position 4 since there are three substitutes at postion 1(the rest one is hydrogen) while there are four substitutes at position 4 .

The imprinted polymer films of 10-CSA and CTH have less rugged morphology (Figure $2 \mathrm{~A}$ and $2 \mathrm{C}$ ) than that of $10-\mathrm{CS}$ (Figure 2B). As expected from the more favorable MIP morphology, ${ }^{11}$ the 10-CS MIP offered better chiral recognition capability than the other MIPs (Table 1).

It should be noted that the $S$-camphor MIP could not resolve camphor enantiomers in any eluent. ${ }^{11}$ The molecular structure of camphor does not have sufficient interaction sites for differential chiral recognition of $(R)$ - and $(S)$-camphor. It is composed of a mostly nonpolar multiply branched cyclic ring with only one polar functional group (carbonyl). Such a structure can hardly fulfill 3-points interaction rule required for good chrial recognition. On the other hand, camphor derivatives of this study include at least one substituent capable of multiple interactions such as hydrogen bonding, electrostatic, steric, or hydrophobic interaction.

Optimization of chiral separation of each MIP. Chromatographic parameters such as organic modifier composition and $\mathrm{pH}$ of eluent were optimized for best chiral resolution of the MIP.

Optimization of acetonitrile composition of eluent: The optimization procedures with respect to acetonitrile composition 
were presented in Figures 3, 4, and 5 for the MIPs with templates of 10-CSA, 10-CS, and CTH, respectively. The recognition properties and selectivities of MIPs are dependent on interactions between the binding cavity and the template enantiomers.

According to Figure 3, chromatographic resolution of 10CSA enatiomers was lost completely when acetonitrile (ACN) content was $80 \%$ or below. ACN content of $92 \%$ offered the best resolution. Further increase of acetonitrile content was avoided since the electrolyte concentration should have been lowered too much owing to solubility limitation. An electrolyte concentration over a certain level is necessary to stabilize $\mathrm{pH}$ and EOF in CEC operation. As a matter of fact, $92 \%$ acetonitrile is hardly used in CEC with a fully filled monolith column. A great advantage of using OT-MIP column is that it can be used with a high organic modifier concentration as well as with a high voltage application as in the present study. On the other hand, it was reported that use of eluents of high acetonitrile content caused frequent formation of bubbles in a full monolith MIP. ${ }^{16}$

The reason why $(1 R)$ - and $(1 S)-10-C S A$ were congested when the ACN content was $80 \%$ or lower can be explained as follows: 10-CSA is a strong acid. It dissociates completely in the $\mathrm{pH}$ range of this study in a highly aqueous eluent to form an anion. The MIP surface is also negatively charged. Electrostatic repulsion between the MIP and the analytes will degrade differential chrial recognition. That is why the chiral separation performance of $(1 S)$-10-CSA MIP was the worst as shown in Table 1. It also explains why the chiral separation resolution of racemic 10-CSA by the BSA immobilized zirconia [13] was better than that of this study. The degree of degradation of differential chiral recognition may be lessened by minimizing the extent of dissociation that can be achieved by decreasing the water content of eleunt (increasing the ACN content).

On the other hand, the trend of differential chiral recognition of 10-CS enantiomers, with respect to ACN comopsition, was totally different (Figure 4). 10-CS is slightly acidic, and hardly dissociates. Thus increasing the water content did not degrade chiral recognition capability, but rather caused enhanced chiral separation together with increased retention times owing to decrease of electro-osmotic flow and reinforced hydrophobic interaction between the stationary phase and the solute. Increased retention was also accompanied by band broadening, thus there should be an optimized ACN content. 70\% Acetonitrile was found the optimum content (Figure 4).

The optimization procedure of chiral separation of the two $\mathrm{CTH}$ enantiomers was given in Figure 5. CTH also hardly dissociates. Nevertheless, the trend of differential chiral recognition of $\mathrm{CTH}$ enantiomers, with respect to $\mathrm{ACN}$ composition, is similar to that of 10-CSA. It should be noted that the optimized conditions are determined by various factors such as absolute and relative change of retention times of two enantiomers, absolute and relative change of their bandwidths, extent of tailing, etc, by varying chromatographic conditions. Details of relevant theories may be very complicated and such discussion is out of the scope of this study. The best resolution for the S-CTH MIP was obtained when the acetonitrile com-

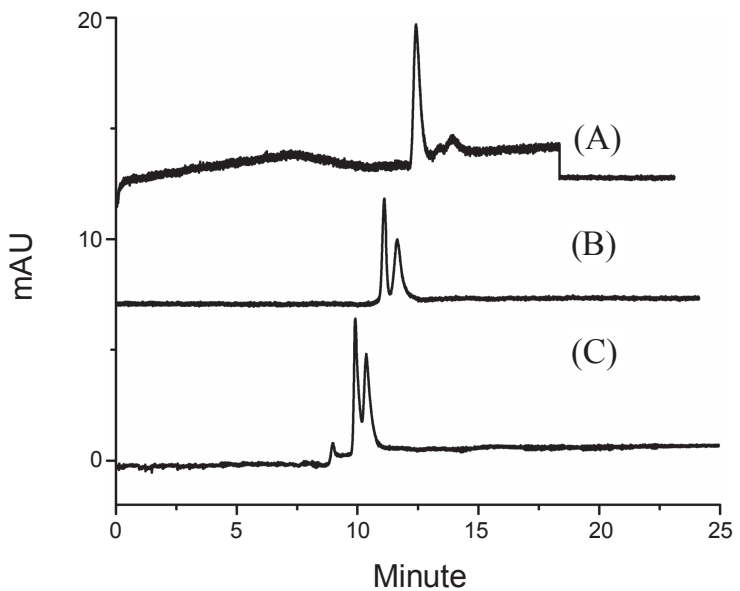

Figure 6. Effect of eluent $\mathrm{pH}$ on separation of racemic 10-camphorsulfonic acid by the $(S)$-10-CSA MIP. (A) pH 2.5, (B) 3.0, (C) 3.5. First peak; (1R)-10-Camphorsulfonic acid, Second peak; (1S)-10Camphorsulfonic acid. Mobile phase: ACN/50 mM sodium dihydrogen phosphate $(92 / 08 \mathrm{v} / \mathrm{v})$. Other experimental conditions are the same as in Figure 3.

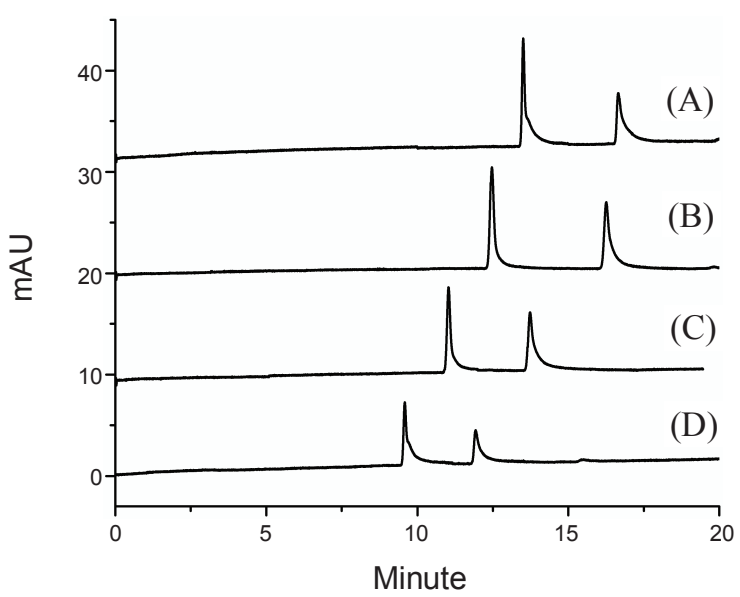

Figure 7. Effect of eluent $\mathrm{pH}$ on separation of racemic 10-camphorsulfonamide by the (S)-10-CS MIP. (A) pH 5.0, (B) 6.0, (C) 7.0, (D) 8.0. First peak; (1R)-10-Camphorsulfonamide, Second peak; (1S)-10-Camphorsulfonamide. Mobile phase: ACN/50mM NaH${ }_{2} \mathrm{PO}_{4} / \mathrm{Na}_{2} \mathrm{HPO}_{4}$ $(70 / 30 \mathrm{v} / \mathrm{v})$. Other conditions are the same as in Figure 3.

position was $92 \%$.

Optimization of eluent pH: The eluent $\mathrm{pH}$ impacts on chiral recognition ability of MIP column. The change in $\mathrm{pH}$ alters the charge density of stationary phase and analytes to subsequently affect the electrophoretic mobility and EOF inside the MIP column.

The $\mathrm{pH}$ optimization procedure of 10-CSA was summarized in Figure 6. It should be noted that both the MIP phase and the analytes are negatively charged. The analytes are completely dissociated in the $\mathrm{pH}$ range considered here (2.5 - 3.5) since 10-CSA is a strong acid. The charge density of MIP is increased with increase of $\mathrm{pH}$. Thus the electroosmotic flow (EOF) was increased with increase of $\mathrm{pH}$, and retention times of analytes were reduced. When the charge density of MIP was increased, electrostatic repulsion between the MIP and the analytes was also enhanced, and chromatographic resolu- 


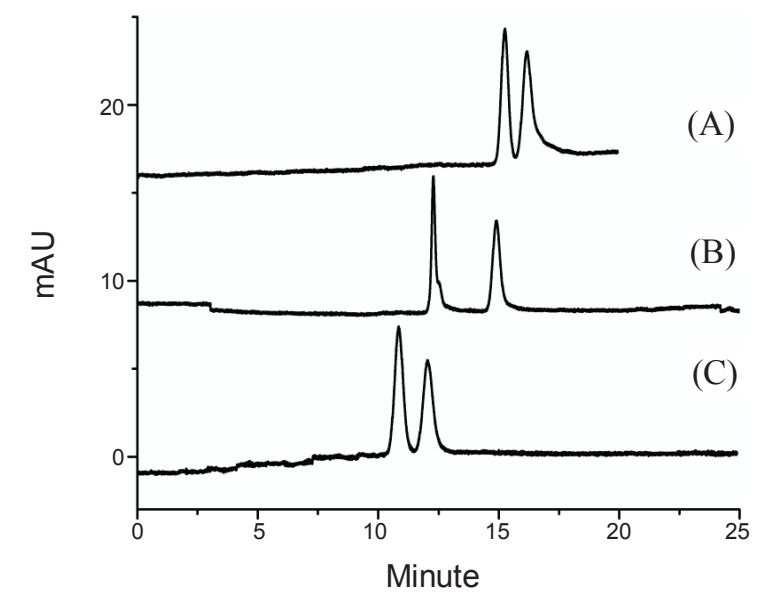

Figure 8. Effect of eluent $\mathrm{pH}$ on separation of racemic camphor- $p$ tosyl hydrazone by the (S)-CTH MIP. (A) pH 6.0, (B) 7.0, (C) 8.0. First peak; $(1 R)$-Camphor- $p$-tosyl hydrazone, Second peak; $(1 S)$ Camphor- $p$-tosyl hydrazone. Mobile phase: ACN/50mM NaH $\mathrm{PO}_{4} /$ $\mathrm{Na}_{2} \mathrm{HPO}_{4}(92 / 8 \mathrm{v} / \mathrm{v})$. Other conditions are the same as in Figure 3.

tion was degraded for the change of $\mathrm{pH}$ from 3.0 to 3.5. When the $\mathrm{pH}$ was changed from 3.0 to 2.5 , the $\mathrm{R}$ - and $\mathrm{S}$ - enantiomers were collapsed to 1 peak. $\mathrm{H}_{3} \mathrm{PO}_{4}$ was used to adjust the $\mathrm{pH}$ of the aqueous $\mathrm{NaH}_{2} \mathrm{PO}_{4}$ buffer. $\mathrm{H}_{3} \mathrm{PO}_{4}$ competes with the analytes for the MIP interaction sites. It seems that the concentration of $\mathrm{H}_{3} \mathrm{PO}_{4}$ was high enough at $\mathrm{pH} 2.5$ to completely degrade the enatioselectivity. Therefore, $\mathrm{pH} 3.0$ was selected as the optimized value for 10-CSA.

While optimizing the eluent $\mathrm{pH}$ for 10-CS (Figure 7), the $\mathrm{pH}$ value was varied from 5.0 to 8.0 since the analytes are slightly acidic. Under the $\mathrm{pH}$ range, the $\mathrm{H}_{3} \mathrm{PO}_{4}$ concentration in the eluent is negiligible, thus collapse of two enantiomer peaks was not observed for decrease of $\mathrm{pH}$. The analytes are almost neutral under the $\mathrm{pH}$ range, thus there was little electrostatic repulsion between the analytes and the MIP and no severe degradation of enantioselectivity was observed with increase of $\mathrm{pH}$, either. The best separation performance was achieved at $\mathrm{pH}$ 6.0.

The optimization of $\mathrm{CTH}$ was investigated in the range of pH 6.0 - 8.0. It is obvious from Figure 8 that the chiral recognition was optimized when the $\mathrm{pH}$ was 7.0.

Table 1 summarizes the results of optimization.

Template structure vs. chiral separation performance of the resultant MIP. The separation performance of the 10-CS MIP was the best among the three MIPs of this study. The poor enantioselectivity of 10-CSA was attributed to the electrostatic repulsion between the analyte and the MIP. However, the superior ennatioselcetivity of 10-CS to that of CTH is yet hard to explain since both templates are almost neutral to give little dissociation in the working $\mathrm{pH}$ range, and are with enough functional groups to fulfill the 3-points interaction rule.

It should be noted that the chrial separation performance of the MIPs in this study is quite satisfactory but it is far inferior to that of MIPs with templates of profen drugs. ${ }^{3,11}$ The chrial center of profen drugs is commonly accompanied with three substitutes- a hydrogen, a methyl group, and a carboxylic acid group. The other substitute is a phenyl group with a bulky sub- stitute. Thus the spatial accessibility to the chrial center of profen drugs is much better than that of the templates of this study.

Spatial accessibility to 10-CS (position 4) is better than that to CTH since the substitute of $10-\mathrm{CS}$ is much smaller than that of CTH as shown in Figure 1. As shown in Figure 2, the morphology of 10-CS is better (more rugged and porous) than that of $\mathrm{CTH}$.

It seems that better stereo-selective interaction between the template and polymerization material is enabled for a template with better spatial accessibility toward the chrial center to result in a MIP of better structure (morphology) and enantioselectivity. There have not been sufficient evidences for such a hypothesis, thus further study is under way.

\section{Conclusion}

In this study, camphor derivatives with a good substituent have been proved to be good templates resulting in a MIP of satisfactory chiral separation performance in contrast to camphor. We have again demonstrated that the general preparation protocol was good enough to secure a thin MIP film inside the capillary innerwall which subsequently offered satisfactory chrial selectivity. The different MIP morphologies were obtained with different templates. The best MIP morphology and enantioselectivity were obtained for 10-camphorsulfonamide.

Acknowledgments. This research was supported by Basic Science Research Program through the National Research Foundation of Korea (NRF) funded by the Ministry of Education, Science and Technology (2010-0015720).

\section{References}

1. Vlatakis, G.; Andersson, L. I.; Muller, R.; Mosbach, K. Nature 1993, 361, 645-647.

2. Klein, J. U.; Whitecombe, M. J.; Mulhollard, F.; Vulfson, E. N. Angew. Chem. Int. Ed. Engl. 1999, 38, 2057-2060.

3. Zaidi, S. A.; Cheong, W. J. J. Chromatogr. A 2009, 1216, $2947-$ 2952.

4. Wulff, G. Angew. Chem. Int. Ed. Engl. 1995, 34, 1812-1832.

5. Sellergren, B.; Shea, K. J. J. Chromatogr. A 1993, 635, 31-49.

6. Schweitz, L.; Andersson, L. I.; Nilsson, S. J. Chromatogr. A 1998, 817, 5-13.

7. Vallano, P. T.; Remcho, V. T. J. Chromatogr. A 2000, 887, 125135.

8. Zaidi, S. A.; Han, K. M.; Kim, S. S.; Hwang, D. G.; Cheong, W. J. J. Sep. Sci. 2009, 32, 996-1001.

9. Nilsson. S.; Schweitz, L.; Petersson, M. Electrophoresis 1997, 18, 884-890.

10. Zaidi, S. A.; Cheong, W. J. Electrophoresis 2009, 30, 1603-1607.

11. Zaidi, S. A.; Han, K. M.; Hwang, D. G.; Cheong, W. J. Electrophoresis 2010, 31, 1-10.

12. The Merck index, An Encyclopedia of Chemicals, Drugs and Biologicals, 12th ed.; 1996; Merck Research Laboratories, Division of Merck \& Co., Inc.: NJ.

13. Park, J. H.; Ryu, J. K.; Park, J. K.; Mcneff, C. V.; Carr, P. W. Chromatographia 2001, 58, 405-408.

14. Kagan, M.; Chlenov, M.; Greenfield, A.; Ho, D. M. J. Chromatogr. A 2006, 1120, 82-88.

15. Gonzalez, G. P.; Hernando, P. F.; Algeria, J. S. D. Anal. Chim. Acta 2006, 557, 179-183.

16. Xu, Y. L.; Liu, Z. S.; Wang, H. F.; Yan, C.; Gao, R. Y. Electrophoresis 2005, 26, 804-811. 